\title{
Study on the environmental design of residential space for elders with dementia
}

\author{
Jinghua Dai ${ }^{1, a}$, Dian Zhou ${ }^{2, b}$, Yishan Xu ${ }^{3, c}$ \\ ${ }^{1,2,3}$ Department of Architecture, Faculty of Human Settlement and Civil Engineering, Xi'an Jiao-tong \\ University, Xi'an, Shaanxi, 710049, China \\ a jhdai325@sina.com, ${ }^{b}$ dian-z@xjtu.edu.cn, cyishan1010@163.com
}

Keywords: Elders with dementia; Residential space environment; Home-style \& small group; Circle-type path; layered care system

Abstract. Along with the aging process, the number of elders with dementia in China is increasing rapidly. However, both the facilities constructed and policies made for them as well as the relevant research and explanation are almost blank so far. Focused on their special physical and psychological status and living demands, this paper proposes corresponding design strategies respectively on environmental atmosphere, care conditions, safety assurance and communication space based on the analysis and summary of relevant cases of residential environment for the aged at home and abroad. Finally a construction model of residential space environment for elders with dementia, which is applicable to the current situation of China, is established and it can be summarized as family-based type(positioning), small group (scale), circle-type road (path), and layered care system (layout).

\section{Introduction}

As one of the common diseases among the elderly, dementia increases significantly with age, which affects $5 \%$ of the population older than 65 and $20-40 \%$ of those older than $85^{[1]}$. However, there lack relevant researches on the policy formulation and institutional arrangements for the elderly and service facilities specially built for them in China for a long time. At present, the old-supporting policies mainly serve for the disabled elders while those with dementia are seldom supported in material assistance, life care and medical services. Therefore, it is urgently expected to make relevant policies and carry out corresponding researches.

\section{Living patterns of elders with dementia}

Ward of elders with dementia, China. In Tongde Hospital of Zhejiang Province in China, relatively independent nursing units are founded by combining the wards with a separate department of senile dementia, which includes 80 beds for elders with moderate to severe dementia. These nursing units are constructed with a single corridor runs in closed management day and night (Fig. 1). It belongs to an attempt of the care for elders with dementia in China. Whereas it is a kind of transformation and mixture in an exploratory stage in China, where there lacks of overall planning for deployment. In fact, plenty of problems are produced with this nursing pattern in daily operation and experience feelings of the elderly as well as the cage-type layout and management.

Green House, United States. In United States, the supporting modes for the aged can be divided into six categories according to their self-care ability and nursing cycle (Fig. 2): Active Adult Community, Independent Living, Assisted Living Facility/Community, Alzheimer's Care, Nursing Home/Skilled Nursing Facility and Continuous Care Retirement Community (CCRC). Among them, the elderly in Alzheimer's care refers to those with memory disorders or Alzheimer's disease who are just the elders with dementia studied in this paper. Aimed at the nursing needs of this group of elders, the concept of "Green House", a non-profit organization, was proposed in 2001, and then it opened in 2003 with the strong support from government. The service type and comprehensive evaluation of the "Green House" are summarized in Table 1. As for this supporting mode for the aged, the home-style nursing is advocated and new family-based design and management structure are adopted, different 
from the traditional professional nursing home. It is aimed to maximally maintain the physical and mental health of the elders as well as their dignity and privacy, which further helps strengthen their independent ability and improve their relations with family members, other elders as well as the caregivers. Based on small units that can be independent or combined together, every unit can accommodate 7 12 elders, while it is composed of the bedroom, dining room, kitchen, bath room and hairdressing salon, etc (Fig. 3) ${ }^{[2]}$.

In addition to building a homey environment, "Green House" also advocated effective use of advanced technologies, such as computer, communication and transportation, which helps increase the nursing efficiency and guarantee the elders' safety. Besides, green spaces are created to help them contact with nature and feel the sunshine. These ideas have been integrated into the planning and design of many communities.
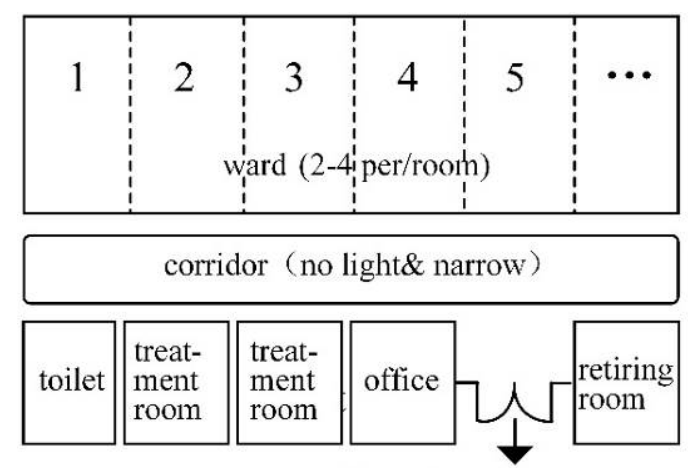

Fully enclosed entrance

Fig. 1 Floor plan of the wards for elders with dementia in Tongde Hospital of Zhejiang Province in China.

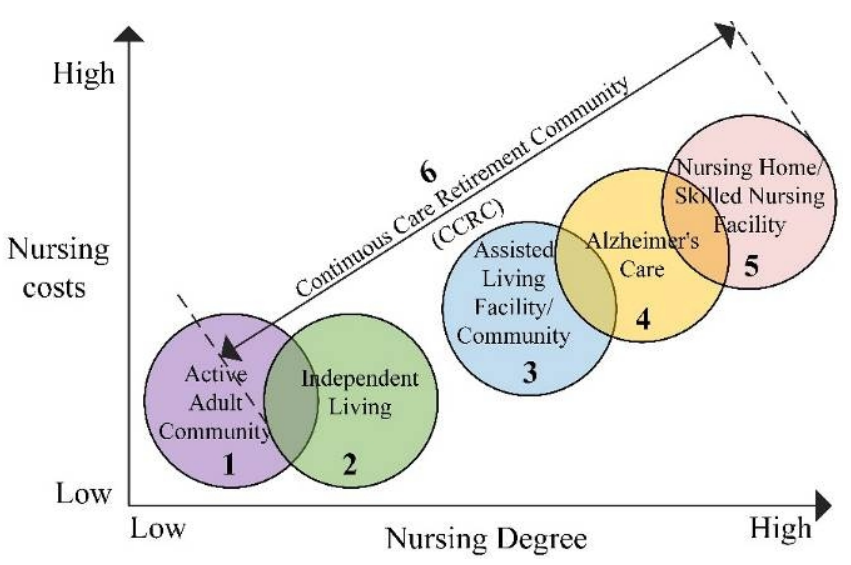

Fig. 2 The six supporting modes for the aged in United States.

Table 1 Type and evaluation of American "Green House" pension model.

\begin{tabular}{|c|c|c|}
\hline Business lines & \multicolumn{2}{|c|}{$\begin{array}{l}\text { home-style, related with the social security and welfare assistance, family } \\
\text { and community are encouraged to participate in. }\end{array}$} \\
\hline Service features & \multicolumn{2}{|c|}{$\begin{array}{l}\text { custodial care, maximally protecting the elders in their autonomy, } \\
\text { dignity, privacy and independence. }\end{array}$} \\
\hline \multirow{6}{*}{$\begin{array}{l}\text { Comprehensive } \\
\text { evaluation }\end{array}$} & $\begin{array}{l}\text { Activities of Daily Living } \\
\text { (dressing, personal care) }\end{array}$ & $\begin{array}{l}\text { Community Services } \\
\text { (laundry, cleaning, ctc.) }\end{array}$ \\
\hline & 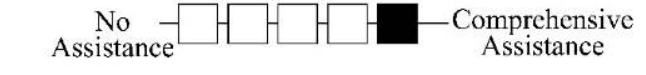 & None - \\
\hline & $\begin{array}{l}\text { Overall Health } \\
\text { (physical, emotional) }\end{array}$ & $\begin{array}{l}\text { Health Services } \\
\text { (medications, nusing care) }\end{array}$ \\
\hline & $\underset{\text { Poor }}{\text { Generally- }} \square \square \square \square-\begin{array}{c}\text { Generally } \\
\text { Good }\end{array}$ & None $-\square \square$ \\
\hline & $\begin{array}{l}\text { Community Activities } \\
\text { (social cvents, outings,golf,ctc.) }\end{array}$ & $\begin{array}{l}\text { Environment } \\
\text { (personal freedom) }\end{array}$ \\
\hline & 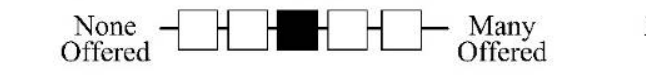 & - Independent \\
\hline
\end{tabular}

Hogewey, Holland. In Dutch, the government set up an elderly supporting village named Hogewey specially for the elders with dementia in Amsterdam. Its internal facilities are the same as normal villages in Holland which include residences, gardens, squares, theaters, post offices, supermarkets and other facilities, and the overall layout is shown in Fig. 4. It is just these scenes close 
to daily life that forms the new nursing environment different from the traditional care model. Many elders with Alzheimer's disease lived here, free from constraint and apparent like healthy people while about 250 medical and nursing staffs lived with them, dressed up as various identities, such as gardeners, customers, cashiers, postmen, etc. Moreover, some furniture and decorations in their memories are specially arranged in the 23 built distinctive houses. 5 7 elders with similar interests share a room tended by 1 or 2 paramedics. In addition, cameras are installed to watch their actions for 24 hours a day to ensure the safety.

The reason why the well-dressed "unusual village" can touch their hearts and make them live a happy and comfortable life is that the village will change their life idea. Rather than regarded as patients who need family and professional care, elders with dementia actually expect more respect and understanding and gently treated.

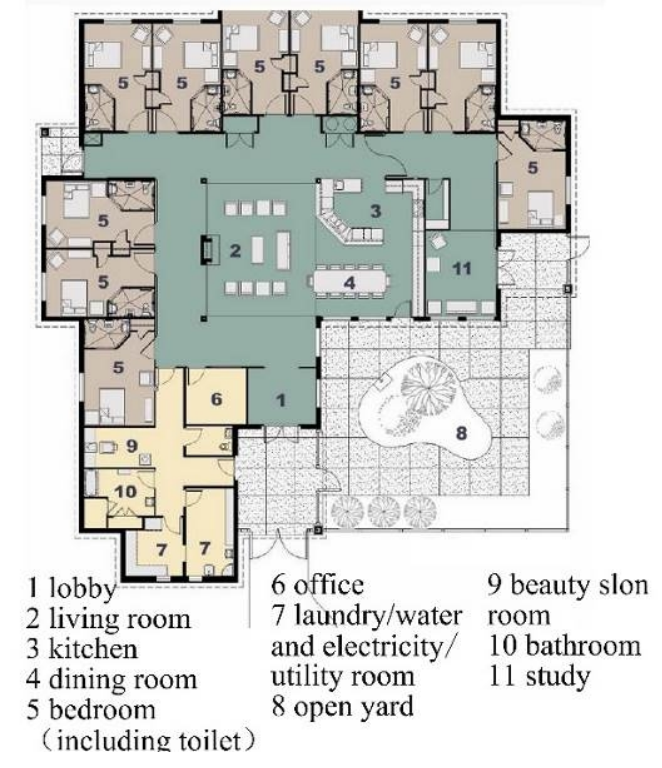

Fig. 3 The floor plan of American "Green House".

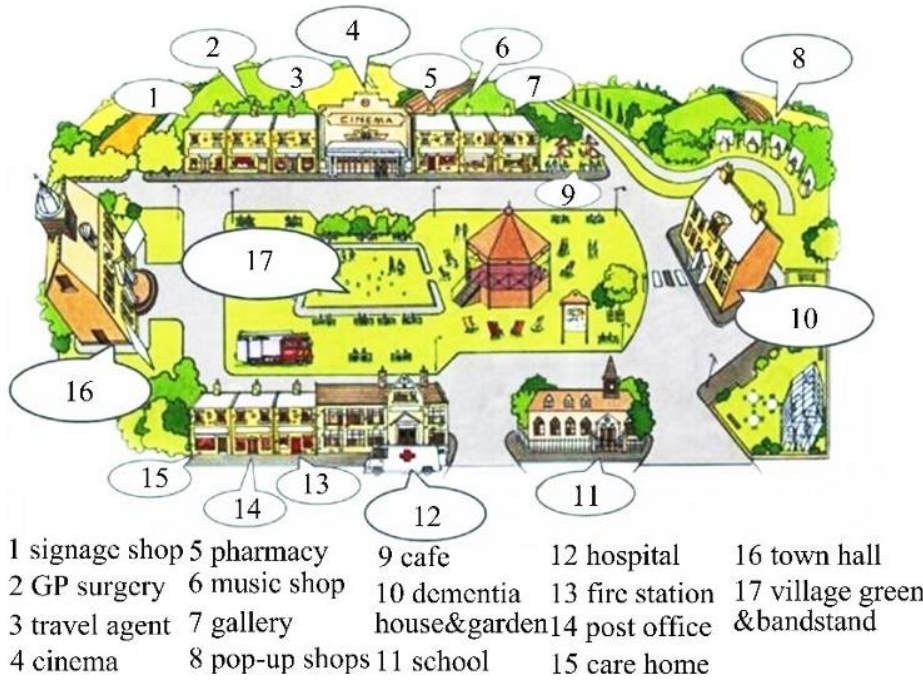

Fig. 4 The overall layout of Dutch Hogewey.

Group Home, Japan. A new type of care service model named "Group Home" (the third type of care models) is developed in Japan based on many existing problems, including that families are unable to provide comprehensive services, care institutions cannot meet their requirements for a home-style life, and population of elders with dementia are continuously increasing. It is aimed to build a pleasant and comfortable homelike environment with the personalized room, family environment, and tailored care service emphasized. Group Home belongs to a kind of small-scale habitation, where every unit can accommodate 5 9 elders, including several major functional spaces like bedroom, living room, dining room, kitchen, public toilet and bathroom (Fig. 5). In order to stabilize the elders' symptoms and help reduce the care cost, nursing workers live with the aged and are responsible for their meals and cleaning services to create a family life in a stable atmosphere ${ }^{[3]}$.

Enlightenment. Through the study and analysis of service facilities constructed for the elders with dementia in the above three countries, United States, Netherlands and Japan, the exploration and research in this field in China can be assessed and guided for a better development. In conclusion, the critical design concepts worthy of learning can be summarized into 4 major points.

Firstly, a homey environment should be established, where the scale, space layout and detailed decoration should be suitable for their daily life. It can not only guarantee the safety and comfort of the elders but also make them feel a strong sense of belonging and intimacy.

Secondly, more chances should be provided for them to contact with nature. It is very helpful for elders to promote the reconstruction of their perceptive function as well as recovery of body function if they can get more touch with blue spaces (blue sky) and green spaces (plants). Get more in touch with 
blue (blue sky) and green (plant) space can promote reconstruction of perceived function in the elderly and recovery of body function.

Thirdly, interaction and communication need be advocated. In this way, the communication between the aged with relatives, other elders and medical personnel will be greatly enhanced and at the same time it helps release the development of dementia.

At last, advanced technologies are taken full advantage of. With intelligent technologies applied, the nursing efficiency will be considerably improved and the elders' safety can also be guaranteed.

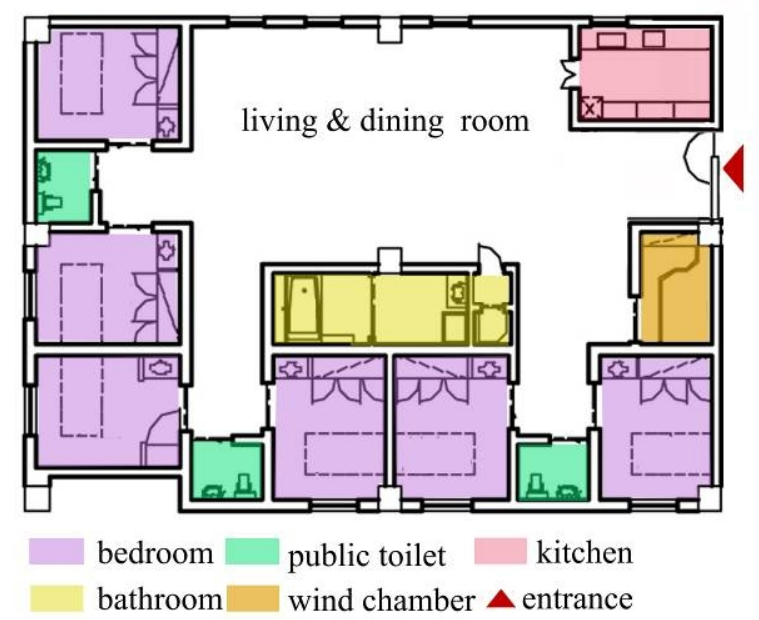

Fig. 5 The plane layout of Group Home in Japan.

\section{Characteristics and residential demands of the elders with dementia}

Dementia is a sort of gradual cognitive function degradation caused by brain injury or disease, the degradation degree of which is fairly higher than that of normal aging. The memory, attention, and language and judgement ability of the elders will be especially affected and they may even cannot identify people, things, time, places, and objects under serious conditions. Dementia can be divided into reversible and irreversible categories according to the etiology, and 90\% of the patients falls into the latter cases and will become more serious. With a very long duration, dementia has a significantly impact on the elderly health while it will additively bring a heavy burden on their family and society.

Elders with dementia often shows a lot of characteristics, including that they cannot recognize their families, may have worse memory and mutable mood, and they will also be confused about time and directions. Also, the symptoms will vary greatly during different periods. Meanwhile, they feel very eager to communicate with others and receive cares from their families. It is easy for them to forget the things recently done or happened while those happened in their younger time are still in their deep memories. Considering their above living characteristics and learning the relevant lessons and experience in developed countries, the residential demand of elders with dementia are concluded into the following four aspects based on the guidance and suggestions in gerontology as well as plenty of investigations and interviews.

Kind and relaxing environment. It is essential to build a familiar living environment for them to receive care and comfort from the family and neighbors, which is helpful to alleviate their restlessness and rejection mood since the spatial cognitive impairment of the dementia elder will become more serious in unfamiliar environment. As for the layout and decoration of the residential space, elders are encouraged to use their familiar items or furniture in order to slow down the development of their memory disorders and at the same time easily remind them of the past memories and thinking ${ }^{[4]}$.

Various and continuous care conditions. The space environment and services adaptive to the dementia elders in different stages should be provided to meet their diverse care demands in different physical conditions and disease severity, which helps avoid the interference between elders of different severity. 
Safety guarantee for activities. The functional decline of elders with dementia becomes increasingly prominent as their cognitive function declines. Therefore it is particularly important to encourage them to take part in appropriate and beneficial activities. Considering that the dementia elders are very easy to get lost or fall, safety precautions should be provided in their living space environment and the differentiation degree of environment is supposed to be strengthened in the design process to help them improve the spatial cognition deficits.

Communication place convenient for participants. Compared with other elders, the aged with dementia needs more participation in social and recreational activities to help them alleviate depressive mood, maintain language ability and strengthen the control of attention. Also their spiritual satisfaction and social identity will be stimulated in this way. Therefore, places and environments beneficial for their communication should be created in the design process if possible.

\section{Key design points of residential space and environment for elders with dementia}

Spatial organization pattern. An appropriate spatial organization pattern should be established based on the practical manpower, and material and financial resources, which mainly includes the following two types.

1) Independent type. It is mainly aimed at the characteristics of the dementia elders that they are not suitable to take part in collective activity of large scale. Care institutes of the independent type generally have a small scale so as to increase the safety and independence of nursing, which as well avoids unnecessary waste of resources.

2) Existing-facility-depending type. For this type, elders with dementia are arranged to live in existing device-aided or nursing-cared units to formulate a group or an area so that professional services can be provided and resources be configured more efficiently for maximum guarantee of the elderly safety ${ }^{[5]}$.

In general, three major design principles should be followed in the construction of residential space. 1) Functionality: rational allocation and definite function sections. 2) Path: simple, straightforward and clear orientation. 3) Layout: safe and convenient principle, and in favor of efficient nursing.

Key design points. According to the physical condition and residential demands of elders with dementia as well as the working characteristics of medical staff and the managers, this paper proposes the following four strategies.

1) Building a kind and relaxing environment atmosphere. At first, a proper scale and functional orientation ought to be established and small family-based group is supposed to be adopted as the major care mode, which is convenient for the nursing staff to be familiar with everyone's preference and characteristics, and further care and manage them. Based on the shortage of nursing assistants in China, every group should contain 10 15 old people and 1 or 2 beds are configured in a room according to the elderly symptoms and degree. In a group except for the bedrooms, public activity space including living rooms and dining rooms, and auxiliary space including kitchens, customized bathrooms and balconies should be set up, which is similar to the functional composition of a dwelling model (Fig. 6). It changes the traditional traverse mode of wards. In addition, the home atmosphere can be further enhanced at entrance of the bedroom and the building, and interactive space with the elderly hobbies and interests as well as their unique memories combined. For instance, an exclusive "memory space" can be set up at entrance of the bedroom to display their representative items or photos (Fig. 7). As for building entrance, the treatment for entrance of housing units that will make the elders feel particularly close and familiar can be referred to instead of traditional public buildings. The public activity space should also adopt the small-scale feature of the home-based care, such as an open kitchen convenient for separate eating, communication and pouring water (Fig. 8), and a balcony that allows 3 5 elders to bask, chat and keep plants. 


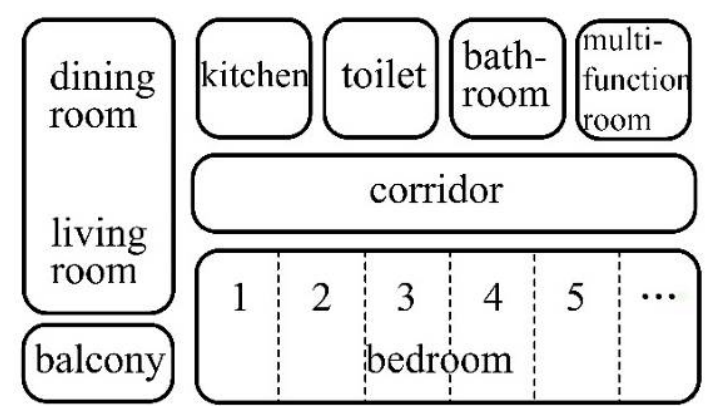

Fig. 6 The functional composition of living space for the dementia elder.

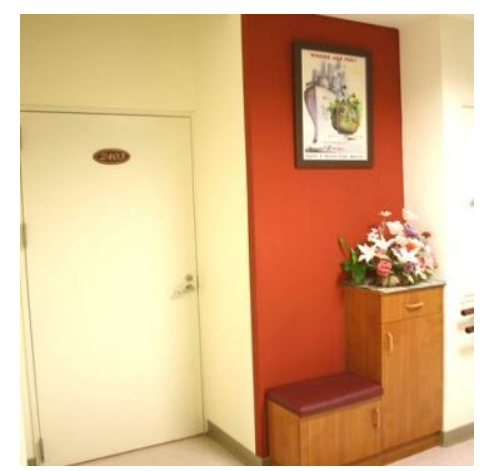

Fig. 7 "Memory space" located at entrance of the bedroom.

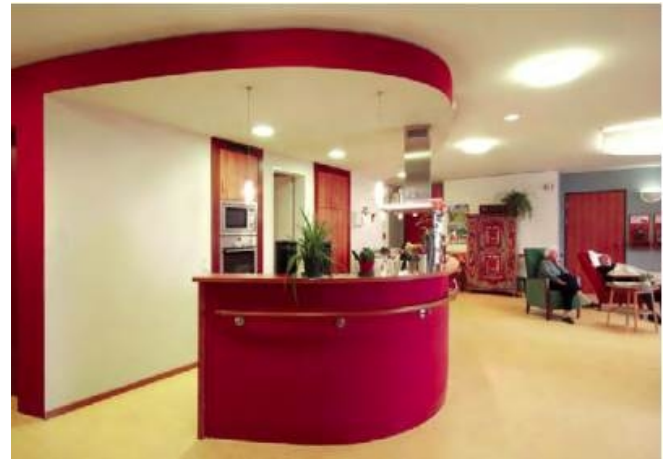

Fig. 8 The open kitchen in public space.

2) Providing multiple and continuous care conditions. The conditions should be configured by layers according to the symptom and degree of the dementia elders. Those of severe, medium and mild degree are respectively arranged from low to high layer and one or more groups can be set up on a layer. Meanwhile, the toilet ought to be reasonably arranged depending on different self-care ability of daily life of the aged. Toilet can be set up in the living room of elders with mild dementia for the purpose of delaying the disease development and strengthening their self-care ability. Considering the decreased ability of self-control of mild and severe dementia, common toilets had better be set up at several places, which not only saves the space of living room, but also is convenient for nursing and the use of elders. Besides, wash-basins can be set up in living room so as to provide convenience for their washing and cleaning and increase the nursing efficiency (Fig. 9) ${ }^{[4]}$.

3) Providing the safety guarantee for activities. Firstly, the convenience and safety of the elderly daily walk should be guaranteed. Many measures can be taken, such as gentle slopes replacing thresholds, steps of equal altitude difference, and setting an intercepting dike at the place dry and water departing to avoid the elderly stumble ${ }^{[4]}$. Also, warning for important entrances (entrances of buildings, living rooms and toilets, etc) ought to be enhanced and distinct markings (such as collision avoidance and block of plants) are utilized to avoid dangerous windows and hidden holes. Clocks, calendars and well-recognized orientation markings can be set on the wall of public corridors to enhance the elderly cognition of time and orientation. Meanwhile the elderly-oriented design of outdoor activity space cannot be negligible for the sake of nursing the aged and prevent obstructing their views, such as continuous and barrier-free roads with explicit direction, appropriate plants including arbors and shrubs. What's more, it is also indispensable that intelligent facilities are configured to guarantee the elderly safety. Therefore, every old people is supposed to wear a GPS 
tracker and every living room should contain an alarm system and ambulation induced device to master the elderly action.

4) Creating the communication place convenient to participate in. Elders with dementia like to walk along one path which should be taken into account in design instead of blindly restricting their freedom. Considering that complicated paths, such as the crossroads and a T-crossing road, easily make them confused and further feel fretted and depressed, a circle-type path should be the best and the most suitable design (Fig. 10). Open public space can also be set up at proper joints, such as projecting portions of corridors, corners and underneath of stairs, to promote their interactive communication and stop and rest, which is also helpful to the nursing workers' care.
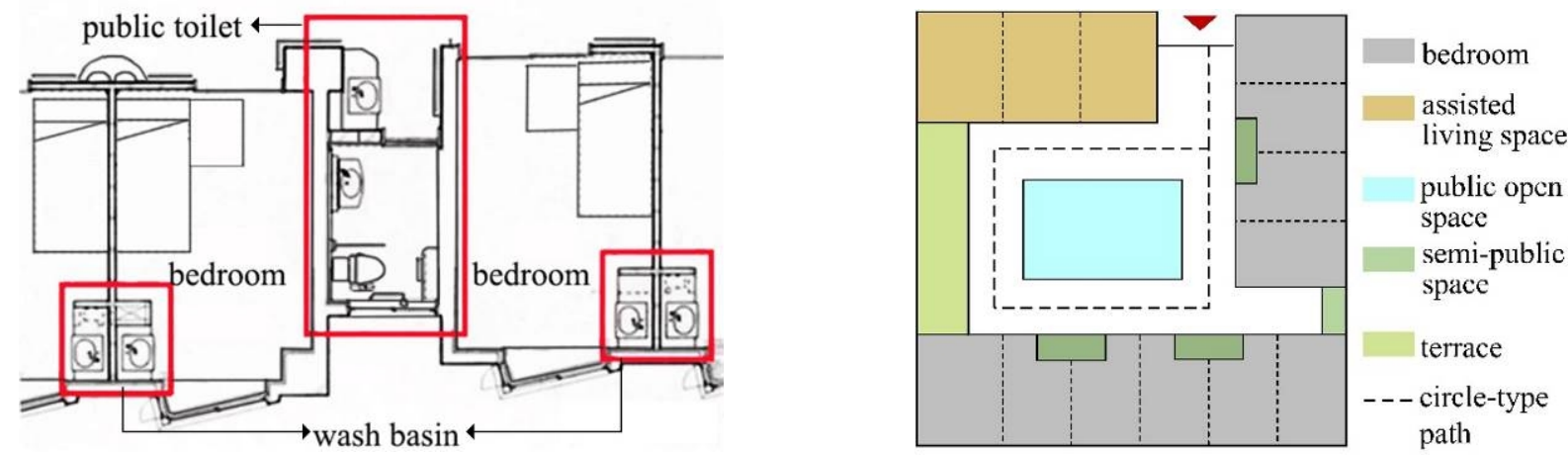

Fig. 9 Settings of public toilet and wash basin. Fig. 10 Circle-type path convenient for the elderly.

\section{Conclusions}

The residential space environment of the dementia elders is closely related to the elderly mental and physical characteristics as well as their residential demands. It also has a lot to do with the medical nursing and operation mode of the nursing institutions. As is known to all, the researches and practices of developed countries in this field are relatively more mature and perfect than that in China, which is worthy of our learning. With China's national condition, the current aging situation and the conception of the elderly support combined, corresponding coping strategies should be proposed based on a fully understanding of the actual demands of elders with the dementia, medical workers and the managers. As a care system and the living space environment based on family-based type, small group, circle-type road, and layered care system are established, it helps maximally maintain the physical and mental health of the dementia elders and meanwhile assists the nursing workers and managers in improving the care quality and increasing the work efficiency.

\section{Acknowledgements}

This work was financially supported by“China Postdoctoral Science Foundation” ( 2015M582665 ) and National Natural Science Foundation of China ( 51278417 )

\section{References}

[1] Sadock, Benjamin James Sadock, Virginia Alcott (2008). Kaplan \& Sadock's concise textbook of clinical psychiatry (3rd ed.). Philadelphia: Wolters Kluwer/Lippincott Williams \& Wilkins. p. 52. ISBN 978-0-7817-8746-8.

[2] Bin Wang: Public hospital as the main agent of institutional elderly care--Junlian nursing home and rehabilitation center for elderly with dementia, Shanghai. Time Architecture, 2012, 06: 64-65

[3] Muqing Hu. "Group Home" is founded in Japan to create home atmosphere [EB/OL]. 
[4] Liyang Shen, Senile dementia care center of Shanghai No.3 elderly home. Architecture Technique, 2014,03: 72-76

[5] Qichao Guo, Fan Yang, Jianxin Sun. Project design analysis and thinking of American dementia care institutions, Proceedings of 2015 Tsinghua aging industry forum, 2015,04.20 